\title{
NOTES ON FLY-WHEELS.
}

BY H. H. BARNES; JR.

The choice of a fly-wheel for reciprocating engines direct connected to alternators is a somewhat serious matter in view of synchronous operation. With prime movers giving a uniform torque, and for belted units, a fly-wheel for the general purpose of obtaining a proper mechanical balance for the operation of the engine is all that is required, and the fact that an alternator has to be driven does not enter into the problem. With directconnected reciprocating engines, however, it is customary to specify a fly-wheel that will limit the angular displacement from the mean position under the worst conditions to a given number of degrees, the limit usually being set at \pm 2.5 electrical degrees. This rule, it has been claimed, gives the best all-round results for the operation of alternating-current machinery. In the following pages an outline has been given of comparative tests of alternators with both light and heavy fly-wheels, the results of which go to show that a good word may also be said for somewhat lighter wheels than the ones to which the above rule leads.

Anyone who has watched the generator instruments in a plant operating alternators in multiple will have noticed a slight periodic swing of the pointers. A closer study of these beats shows there are two distinct varieties. Usually the swing is in step with the revolutions of the engine and is entirely independent of the field excitation of the alternator. In other cases the beats have a distinct period of their own that varies with the field excitation and consequently with the bus-bar pressure. In the first case the variation in the driving torque of the engine, which repeats itself periodically with each revolution, imposes itself upon the alternators and gives forced oscillations. The 
variable period, on the other hand, is that of natural oscillation. Natural oscillations invariably appear when an alternator is paralleled a little out of step, and under some other conditions as well, and occasionally even predominate to the exclusion of forced oscillations. At all events, it is an easy matter to determine the natural period experimentally. Its importance and its intimate relation to fly-wheel capacity will be more thoroughly appreciated if we analyze the results obtained in three different plants in which alternators of identical design are direct connected to cross-compound reciprocating engines. The interesting feature in these plants and the reason for singling them out being that, while the generators are identical and the engines. nearly so, the fly-wheels differ very considerably in weight.

The generators throughout are 500-kw., two-phase, $100 \mathrm{rev}$. per min., 60-cycle units of the inductor type. Their short-circuit. ratio with no-load excitation is 3.5.

The following table gives the fly-wheel data:

\begin{tabular}{c|c|c|c|c|c}
\hline & \multicolumn{2}{|c|}{ Fly-Wheel. } & \multicolumn{3}{|c}{ Moment of Inertia in lbs. x (ft.)2 } \\
\hline Plant. & $\begin{array}{c}\text { Weight of rim } \\
\text { in lb. }\end{array}$ & $\begin{array}{c}\text { Outside diam- } \\
\text { eter in feet. }\end{array}$ & Fly-wheel. & $\begin{array}{c}\text { Revolving element } \\
\text { of alternator. }\end{array}$ & Total. \\
\hline A & 30000 & 15 & 46150 & 16900 & 63050 \\
B & 50000 & 16 & 92430 & 16900 & 109330 \\
C & 60000 & 18 & 143500 & 16900 & 160400 \\
\hline
\end{tabular}

The natural frequencies obtained experimentally for each plant at no load are shown in Fig. 1 on next page.

It will be noted that the units with the lighter wheels have the higher natural frequency, as was to be expected. The effect of this difference in natural frequency upon the behavior of the engine-governors is marked. With the governor dash-pots in the condition in which the engine builders left them, parallel operation at normal pressure was a failure in all three plants. Upon throwing two units together, cross-currents would build up, and eventually the surging would become so violent that the machines would have to be separated. The frequency of surging was that of natural oscillation throughout. With the same setting of the engine dash-pots, however, the machines in $C$ would run together acceptably at no load at pressures of $60 \%$ and less of normal, and the machines in $B$ at pressures of $40 \%$ and 
Jess of normal, whereas those in $A$ failed to operate satisfactorily at $50 \%$ of normal, which was the lowest pressure tried.

By artificially decreasing the oscillations of the governors, by means of the dash-pots, the units in $A$ could be made to operate well at normal and higher pressures. The units in $B$ showed an improvement at pressures considerably above normal (tests at reduced pressures had to be omitted here to avoid interfering with the commercial operation of the plant), while no improvement whatever was noticeable in $C$, except for the lower pressures. The former average limit of $60 \%$ of normal being extended to over $80 \%$.

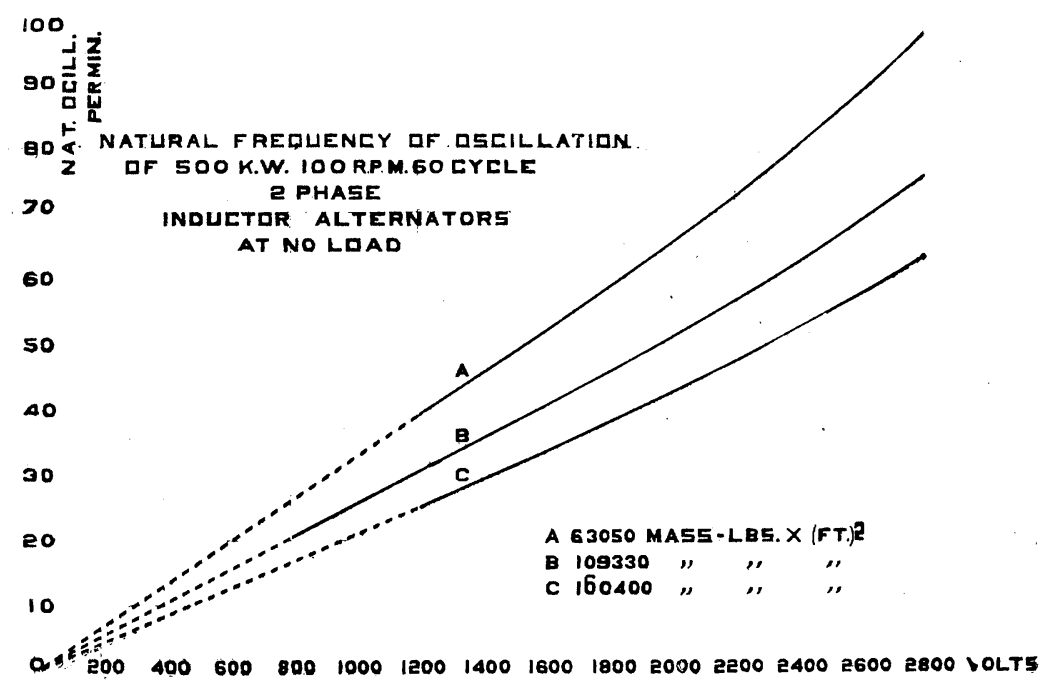

Fig 1.

A still further increase in the damping of the governors in $C$, however, resulted in a decrease in the limiting pressure, until with completely-blocked governors parallel operation became impossible at any and all pressures. This result and similar ones obtained elsewhere would indicate, it seems, the existence of an inherent tendency toward cumulative surging that is in no way connected with the behavior of the engine governors.

The origin and nature of this tendency are not directly apparent. It seems likely that they are intimately connected with the armature reaction of the alternators, but whether a departure from a uniform turning moment in the prime mover is also essential to its appearance may be doubted. As far as the writer is aware, there is no indication in dynamics in the 
theory of forced and natural oscillations of a possibility of forced oscillations producing cumulative surging. If this is so, it might be imagined, although there is no direct experimental evidence to support the idea, that the surging which so frequently appears and is at times so hard to control, in alternators operating in multifle (to limit ourselves to a definite case) is due, not to the lack of uniformity in the turning moment, but rather to the effect of heary wheels upon armature reaction phenomena. It is also probable that we have operating, in addition to this, causes of the same nature as those that produce cumulative surging in a Watt governor system. It should be clearly understood that this is hardly more than a guess. But it is one that would be worth while checking up by making the following test. for which the writer has unfortunately not had an opportunity.

In a plant in which direct-connected alternators are given to chronic cumulative surging that will not disappear even when running on the throttle with governors blocked, disconnect the engines and drive the alternators by means of individual constantcurrent motors. If we parallel the alternators under these conditions and surging still persists, we will have eliminated from the problem the angular variation of the engine. If it should fail to appear, however, the origin of the trouble would be definitely located in the engine. Whatever the origin of this inherent tendency to surge may be we rinust more than counteract its effect to insure successful parallel running, a result that may be brought about in two ways: by using heavy wheels-or else by operating at reduced pressures-the natural period of the unit may be lengthened to such an extent that the engine governor, instead of adding to the disturbance will have time to grapple with any surging that may appear, and wipe it out. An example of this is the behavior of the machines in plants $B$ and $C$ at reduced pressures. This solution has the drawback of leading to wheels of prohibitive weight for slow-speed anits, and furthermore, of making the successful operation of the plant dependent to a certain extent upon the adjustment of the engine governors.

The second and better way to overcome the inhorent tendency to surge is to provide sufficient electrical damping. Any oscillations that may appear will then be taken care of by the damping, and the assistance of the governor will no longer be required. In fact the damping action will be more rapid and final if the governor dash-pot is adjusted so that the governor will not respond to natural oscillations, as, in the majority of cases, it 
would only strive to maintain the latter if left free to act. As it is easier to adjust the dash-pot with this end in view, to bridge over a short period than a long one, we see that light wheels with their short periods are more desirable in this respect than heavy ones. This argument may seem weak at first when applied to plants in which forced oscillations predominate, but experience teaches that the disturbing influence of the natural period is felt, even when there is no immediate indication of its existence on any of the switchboard instruments. More delicate instruments than the ones usually used would probably reveal the natural period, and even with instruments which appear at first to indicate forced oscillations only, a battle for supremacy between the forced and natural periods may frequently be detected by scrutinizing the movements of the pointers with more than usual care.

To bring out a little more clearly the effect of a change in weight of wheel upon the behavior of the engine governor, we might say that with very heavy wheels and consequently long natural periods the governor is able to kill off unaided any surging that may attempt to develop. With very light wheels, on the other hand, the governor is barely affected by surging and the task of holding it down devolves upon the electrical damping of the units. There is an intermediate zone between these two extremes in which the governor would directly assist the tendency to surge if it were not prevented from doing so by special adjustment of its dash-pot. If such adjustment is not made, it is evident that what electrical damping there is will be more sorely taxed, and the heavier the wheel the more likely it will be that the damping will be unequal to the task of carrying this addititional burden and that cumulative surging will set in.

If neither of the conditions we have been discussing is met, as in plants $B$ and $C$ at normal voltage, successful running may be said to be out of the question. The best remedy for the cumulative surging that will undoubtedly set in in such cases is to increase the electrical damping of the machines, until it is able to hold in check the inherent tendency to surge. This was done in the above plants with eminently satisfactory results.

It may also be pointed out that the successful running at. normal pressure with dampened governors in plant $A$, compared with the failure to run in plants $B$ and $C$, would indicate that the relative increase in electrical damping is more rapid than the corresponding increase in the inherent tendency to surge for a given decrease in weight of fly-wheel. Should this be corroborated. 
by similar observations elsewhere, a further most weighty argument would be added in favor of light wheels. However this may be, the tests already described, and they are but a few of those that could be cited, lead to the conclusion that a decrease in fly-wheel capacity is not synonymous with an increased tendency to surge on the part of alternators. The following observations will emphasize this point still further.

In plants $A$ and $B$ individual exciters are belt driven from the shaft of each alternator, and facilities for paralleling the exciters are provided, besides the usual switches. With this arrangement, if two alternators are paralleled while energized by their own separate exciters, and the pressure and dash-pot conditions are such that cumulative surging sets in, the surging may be very rapidly stopped at all loads by simply paralleling the exciters. Tests were made to ascertain the nature of this action of the exciters. The results proved that it is directly comparable to that of electrical or viscous damping and not related to the constancy of the exciter pressure.

Other conditions being the same, it is reasonable to assume that the time taken to dampen out cross-currents by paralleling the exciters would give a fair indication of the relative tendency to surge existing in these two plants. Actually, the average time required is about the same in both plants, with possibly a slightly quicker action $\mathrm{in} A$. From which the conclusion must again be drawn that a decrease in fly-wheel capacity and consequent, increase in angular variation while running does not decrease the stability of the generators. In making these tests it was noticed that individual results in $A$ were affected to a very slight extent by the relative position of the driving cranks of the engines. In $B$ and $C$ and numerous other plants experimented upon, this influence, if it existed at all, was so slight that it was impossible to distinguish it from that due to other causes.

Passing on to another phase of the subject, we find that the limitation usually imposed upon the maximum angular variation of alternators is intended, among other things, to limit the cross-currents due to this variation. Thus, for a variation of \pm 2.5 electrical degrees and a short-circuit ratio of 2.5 , the cross-current will amount to approximately $10 \%$ of the fullload armature current, and no more. If we consider, however, that a $10 \%$ current results in an $I^{2} R$ loss of less than $1 \%$ of normal, and that the normal $I^{2} R$ armature loss is itself but a fraction of the total loss in the generator, we realize that at least a $25 \%$ cross- 
current may be permitted without fear of appreciably increasing the total losses. Especially as such cross-currents would appear under the most unfavorable conditions of load and cut-off only, the average exchange of current during the day's run undoubtedly remaining well below the maximum. It might be well to add that there should be not any trouble from blinking of the lights if the cross currents do not exceed the value mentioned. There do not appear to be any very good reasons, therefore, why objections should be raised on this score to the use of lighter fly-wheels giving a considerably great variation than the customary 2.5 degrees.

We shall now examine the effect of a light wheel on the generating units upon the behavior of synchronous apparatus. We have in $C$ a good example of a plant working ander these conditions. Exceptionally light wheels are in use there and numerous synchronous motors and rotaries are in operation on the system. Not a trace of trouble has developed in this plant since the start, however, notwithstanding the light wheels.

Some interesting experiments that are very much to the point were made recently by Mr. P. C. Oscanyan. In a plant equipped with 850-kw., 60-cycle, 90 rev. per min. alternators, direct connected to cross-compound reciprocating engines, the fly-wheels were designed to maintain the angular variation within \pm 2 degrees of mean. The rims of the wheels were laminated and weighed normally $1000001 \mathrm{lss}$. With a machine with less than onequarter of its rim in place running singly and in parallel with a completed unit, no difference whatever could be detected between the behavior of the incomplete machine and that of its neighbors. It would operate in parallel under all conditions of load, and when running singly would carry a load of synchronous converters without any indication of trouble. On the strength of this, and without going further, it may be said that surging and allied troubles in synchronous apparatus are not necessarily associated with light wheels and increased angular displacement. They are due, it would appear, more to certain imperfectly understood relations between the number of generating and receiving units, their moments of inertia, output, speed, etc., than to the existence of a more or less pronounced angular variation dependent upon the fly-wheels.

The question of electromechanical resonance remains as being about the only possible further source of trouble that might be introduced by a change in weight of wheel. Resonance, it may 
be added by way of explanation, is said to exist in a system when the ratio of forced and natural frequencies is nearly or exactly equal to unity; its effect is a tremendous amplification of the original forced oscillation.

By referring to the curves of natural frequencies on page 355 , we see that the normal natural frequency of the units in plant $A$ is 81 per minute. The forced frequency determined by the revolutions is 100 . By forcing the excitation until we reach pressures and consequently natural frequencies, that are considerably above normal, we might expect to see the effects of approaching resonance. In reality, noticeable cross-currents begin to appear at a frequency of 87 ; at 90 they are already quite pronounced. They are not cumulative, however (provided the engine dash-pots are in order), but increase and decrease periodically with a main frequency equal to the difference between the forced and natural frequencies. The slip, as it were, between the two frequencies may be counted in this way with great precision. We have here the phenomenon of interference, which appears whenever the damping of a system is very weak. If we increase the damping in this case, as, for instance, by paralleling the exciters, the main period disappears, and the cross-currents take on an average value with a regular but slight beat of forced frequency. A further increase in natural frequency runs up the cross-currents very rapidly until they are far in excess of the full-load armature current for a ratio of frequencies nearly equal to unity. These results show clearly enough that resonance is a real and very serious danger, and one to be avoided with the utmost care, as there is no acceptable way of overcoming it when encountered in practice. In order to avoid it, we should have a formula for predetermining the fly-wheel and the corresponding natural period, which we could then make as different as we choose from the forced period, already determined $b_{s} y$ the revolutions of the engine.

The oldest and at the same time the best formula for this purpose that the writer knows of, was published by Paul Boucherot in 1892, in Vol. 45, issue No. 31 and 32 of La Lumiere Electrique. It is, with a recent slight modification:

$$
T=0.31 N \sqrt{\frac{M}{f \times E \times I_{\mathrm{e}} \times p}}
$$

where

$T=$ Natural period of oscillation in seconds at no load. 
$N=$ Revolutions per minute.

$M=$ Total moment of inertia of all rotating parts (fly-wheel, crank discs, inductor or revolving field, etc), expressed in $1 \mathrm{~b} . \times(\mathrm{ft} .)^{2}$.

$f=$ Electrical frequency per second.

$E=$ Effective e.m.f. per phase or leg.

$I_{\mathrm{e}}=$ Effective short-circuit armature current corresponding to $E$.

$p=$ Number of phases.

The greatest discrepancy that has thus far appeared in checking this formula experimentally in nearly a dozen plants is one of $+6 \%$, the calculated period being that much longer than the observed one. The mean error for all the plants was $+3 \%$. The reliability of the formula for practical purposes is therefore beyond doubt.

With this formula to assist us, it is an easy matter to predetermine a fly-wheel that will at least avoid resonance once we have agreed upon the closest ratio permissible between the forced and natural frequencies. The choice of this ratio depends upon numerous considerations. In the first place we have, as the essence of resonance, the increase in angular variation that is bound to appear after paralleling, even when absolute resonance is still quite remote. There is also the possible error between the calculated and the actual fly-wheel, the rather doubtful values that are sometimes used for the short-circuit current and the increase in natural frequency with the load and with a decreasing power-factor. Making due allowance for these various factors, let us assume for the moment a natural frequency at no load equal to $70 \%$ of the forced.

If we introduce this condition into the formula on the preceding page, and substitute $P \times k$ for $E \times I_{\mathrm{e}} \times p$, and $W r^{2}$ for $M$ wherein $P$ is the normal output of the alternator in kilowatts, $k$, the ratio between the short circuit and full-load armature current, $W$ the weight in $1 \mathrm{~b}$. and $r$ the radius of gyration in feet of all the revolving parts combined, we get

$$
W r^{2}=25^{P \times f \times k}
$$

It remains to be seen if a wheel based on this formula is sufficiently heavy to keep the cross-currents within bounds. It appears upon examination that for such a wheel the maximum cross-current between alternators driven by any average cross- 
compound steam-engine would not exseed $25 \%$ of the full-load armature current, even under the worst conditions, which may be considered an entirely permissible figure.

For an ordinary cross-compound unit and a short-circuit ratio of $k=2.5$, the maximum angular variation may be assumed to be within \pm three electrical degrees of mean when running singly. With a $70 \%$ ratio of frequencies, however, the angular variation of a unit running singly may be practically doubled upon paralleling it in with its fellows, and it is upon this increased variation that the calculation of the cross-currents has been based. With alternators with a higher short-circuit ratio and therefore better regulation, the angular variation will be less on account of the heavier fly-wheel called for by the formula. The cross-currents, on the other hand, remain the same for a given type of engine rerardless of the regulation of the alternators. With single-crank engines these currents will be somewhat heavier than with cross-compound engines, and with multi-cylinder engines a trifle less. The difference in the latter case will hardly be sufficient to demand a separate formula, and the same may be said of vertical as compared with horizontal types of engines. With single-crank engines, however, the maximum angular variation should be determined and if the design of the engine is such that the variation in a single engine materially exceeds \pm three electrical degrees, it would be advisable to modify the constant in the formula to a slight extent.

While there are apparently no disadvantages connected with the use of relatively light wheels, there are, at least, two distinct advantages that should not be overlooked. We have already seen that a light wheel means a high natural frequency for which it is relatively easy to get the engine governors to behave properly. In practice this is a very real and important advantage, and one not to be lightly dismissed.

$\Lambda$ considerable saving in first cost may also be affected by using lighter wheels, especially in the case of slow-speed units, where the amount of iron that may be saved in the fly-wheel foots up to quite a respectable sum At the same time the general wear and tear upon the bearings of the engines are lessened.

We believe, therefore, that the formula given above may be used with perfect safety in predetermining fly-wheels for directconnected steam-engine drive, with the following exceptions. For high speeds the wheels obtained, while meeting all electrical requirements, would hardly be heavy enough to give the engine a proper mechanical balance, and in such cases 
heavier wheels, as determined by the engine builders, should be used. Variations of the formula will also suggest themselves in individual cases. Thus, for instance, with engines giving an exceptionally even driving torque connected to alternators of excellent regulation, the permissible ratio between natural and forced frequencies might be advanced to 0.75 or even more without serious risk of encountering resonance phenomena or of introducing troubles of other origin. With single-crank engines, on the contrary, it will occasionally be necessary to use a somewhat heavier wheel than the one indicated by the formula.

To sum up our main conclusions, we may say that the flywheel-as distinct from the assistance given either by the engine governor or the damping of the unit-is impotent to ensure satisfactory synchronous operation. Except for these two influences, the inherent tendency toward cumulative surging existing in the unit would predominate, and parallel running would be impossible

It also seems advisable to use a somewhat lighter fly-wheel than would be considered conservative practice to-day. This greatly facilitates the adjustment of the engine governors while it does not decrease the stability of the system. For it would appear that the electrical damping of the units (which varies with their natural period and consequently with the fly-wheel) at least retains its relative strength as compared with the inherent tendency to surge for wide variations in weight of wheel. Therefore, if the damping is sufficient to hold the tendency to surge in check when a heavy wheel is "sed, it remains so for a light wheel. This argument applies to a change in weight of wheel for a given generating unit only, and should not be construed as one in favor of increased angular variation as such.

We also find that electromechanical resonance is a serious danger that must be avoided by making a proper choice of flywheel. A whel selected with this end in view is at the same time satisfactory for general operating purposcs, provided there is sufficient damping in the unit to check the inherent tendency to surge.

If we inquire, in concluding, what fly-wheel capacity suggests itself for direct connection to gas-engines, it must be said that it would be a little venturesome to advance a cut and dried rule at present in view of the limited practical experience with this type of prime mover. By analogy with steam-engine experience, however, it may be said that light fly-wheels and vigorous electrical damping is the combination that is most likely to win out for this class of work

[For discussion on this paper, see page 461.] 